\title{
Une écriture implacable : présences du politique dans l'œuvre de Pascale Kramer
}

\author{
Ursula BÄHLER \\ Université de Zurich
}

\begin{abstract}
L'analyse proposée de L'Implacable Brutalité du réveil et d'Autopsie d'un père permettra de sonder la place et l'impact de la politique et du politique dans les romans et la réflexion littéraire de Pascale Kramer.

Keywords : Pascale Kramer, roman contemporain, roman francophone, roman et politique
\end{abstract}

Jusqu'à quand alors pourrai-je encore me permettre de refuser de choisir un ennemi?

Pascale Kramer (20I9 : 5I)

Les romans de Pascale Kramer ne se lisent pas d'emblée comme des textes politiques au sens traditionnel du terme, entendu comme une forme de réflexion sur les affaires de la cité. À y regarder de près, on constate, cependant, que dès le début, l'univers kramerien contient des éléments qu'on qualifiera bien de politiques dans ce sens précis, restreint du mot. La présence de ces éléments se décline de trois manières, essentiellement. Il y a, d'abord, des romans dans lesquels une problématique socio-politique se perçoit à la façon d'un basso continuo, d'une atmosphère générale qui s'installe à la lecture et se caractérise par un malaise grandissant. Citons comme exemple Retour d'Uruguay (2003), où le lecteur sent beaucoup plus qu'il ne le sait qu'il a aussi affaire à une histoire de violence domestique et d'affaires immobilières aux limites de la légalité. Il y a, ensuite, des textes qui évoquent explicitement des problèmes politiques sans les mettre pour autant sur le devant de la scène, comme c'est le cas d'Un homme ébranlé (20II) qui évoque à plusieurs reprises des violences dans les banlieues et les entrelace, mais de manière plutôt lâche, à l'histoire de la maladie de Claude. $\mathrm{Et}$, finalement, il y des textes dans lesquels la politique forme une partie essentielle de l'intrigue. L'Implacable Brutalité du réveil (2009) et, plus encore, Autopsie d'un père (2016) sont de ceux-là, le premier parlant de la guerre des États-Unis en Irak, le deuxième d'un personnage qu'on qualifierait de 'nouveau réactionnaire'. On constate, sur l'ensemble de la production romanesque de Pascale Kramer, une évolution vers un impact grandissant de la politique, qu'on pourrait mettre en rapport avec des publications récentes 
qui témoignent d'un engagement social et intellectuel de l'auteure, telles Chronique d'un lieu en partage (20I7) et la contribution à un volume sur Carl Spitteler, intitulé Helvétique équilibre (2019)․․ Le dernier roman en date, cependant, Une famille (20I8), ne renvoie à la réalité politique stricto sensu qu'à travers une allusion au mariage pour tous. Cet exemple nous met utilement en garde contre la tentation d'une lecture téléologique de l'œuvre de Pascale Kramer. S'il y a bien une tendance globale vers un intérêt accru pour des questions politiques dans ses romans, on n'y verra pas une nécessité, ni, encore moins, faut-il le souligner, un critère d'évaluation.

Dans son discours tenu lors de la remise du Grand Prix suisse de littérature $20 I 7$ à Pascale Kramer, Alain Berset a mis l'accent sur le côté politique de l'œuvre de la lauréate : est politique, pour lui, l'empathie, notamment, que l'écrivaine porte à ses personnages, "gens normaux ", sans les juger, mais en leur donnant une place, une voix (Berset 2017). Le mot politique prend un sens plus vaste ici : il désigne des vertus humaines, l'empathie en l'occurrence, considérées comme souhaitables sinon indispensables à un comportement civique au service de la cohésion sociale et du vivre ensemble. C'est dans ce sens aussi que raisonnent en général les philosophes qui mettent en évidence les valeurs éthiques de la littérature, que ce soit dans une perspective herméneutique, comme Paul Ricœur, néo-aristotélicienne, comme Martha Nussbaum, ou pragmatique, comme Richard Rorty, pour ne nommer ici que trois représentants majeurs et fondateurs de ce courant philosophico-littéraire ${ }^{2}$.

Dans les pages qui suivent nous essaierons de voir comment les deux types de présence du politique que nous venons d'esquisser, ancré, l'un, dans la réalité événementielle, et relevant, l'autre, d'une réflexion axiologique et morale, sont liés dans l'univers romanesque de Pascale Kramer, en nous penchant sur L'Implacable Brutalité du réveil et Autopsie d'un père.

\section{Devenir adulte}

L'Implacable Brutalité du réveil, dont l'action se situe en Californie, le temps d'un été, est construit sur la mise en scène de deux drames parallèles fondés tous deux sur une tension entre paraître et être, idéalisation et réalité. Le premier s'articule sur un versant familial, le deuxième s'inscrit dans une dimension sociale. Si le drame familial est le principe structurant de presque tous les romans de Pascale Kramer ${ }^{3}$, au point que le titre du dernier

I On pourrait citer également son texte «Salauds de pauvres! L'effroi et la honte » (2016).

2 Voir Bähler \& Klinkert (2016) et Bähler \& Fröhlicher (2019), ainsi que, ici même, la contribution d'Anne-Frédérique Schläpfer, pp. 33-45.

3 Voir aussi Bähler (20II). 
en date, Une famille, pourrait bien s'appliquer à l'œuvre dans son ensemble, L'Implacable Brutalité du réveil est le premier livre dans lequel un drame social, et plus précisément politique, la guerre des États-Unis en Irak, en l'occurrence, prend de l'ampleur pour former un véritable deuxième volet, à la fois homologue et complémentaire au premier.

Le drame familial se noue autour de la protagoniste Alissa, et c'est dans la perspective de celle-ci que l'essentiel du récit nous est narré : jeune et belle femme âgée de 27 ans, issue d'un milieu aisé, diplômée d'université, mariée depuis peu de temps à Richard, jeune homme tout aussi attrayant, elle vient d'accoucher d'une petite fille, Una. Cette naissance constitue un 'réveil brutal' à la réalité, elle marque un ébranlement radical de l'identité d'Alissa et de sa façon de voir le monde. Incapable d'assumer pleinement et de manière instinctive la vie de son enfant - le nom générique d' "Una » exprime bien ce (non) rapport -, la protagoniste se voit ballottée entre rejet, indifférence, jalousie et tendresse. La fin du roman reste ouverte quant à l'attitude qu'adoptera finalement Alissa vis-à-vis de son bébé.

On peut lire l'histoire d'Alissa comme celle d'une dépression post-natale somme toute 'classique', ce qui, soulignons-le ne signifie pas banale ; mais, et c'est cette piste que nous emprunterons ici, on peut la lire également comme une version contemporaine du bovarysme ${ }^{4}$. Tout au long de son enfance et de sa jeunesse, son entourage, à commencer par ses parents (et avant tout sa mère), relayés par ses amis, ont fait croire à Alissa, que cela soit volontairement ou involontairement, qu'elle aura un avenir extraordinaire, une vie de bonheur parfait, idéal auquel elle s'est complètement identifiées. Confrontée à la réalité de sa vie de jeune maman, cet idéal se fissure, ce dont elle (se) rend compte de manière toujours plus lucide au cours du récit :

4 Les parallèles entre Emma Bovary et Alissa sont en effet nombreux et mériteraient une étude particulière. Quelques citations choisies au fil de la lecture suffiront ici à montrer la proximité des perceptions de la réalité par les deux protagonistes, la première interprétant le monde à travers ses lectures de jeunesse, la deuxième à travers les images suscitées par les discours de ses proches. Chez Kramer : «Elle [Alissa] ignorait quel défi elle se lançait, à quelle tyrannie ou hypocrisie elle se soumettait, mais il était exclu qu'elle renonce au bonheur anticipé, qui restait promis ou dû, ou du moins désiré avec une telle crédulité qu'il lui était impossible de s'avouer son insatisfaction » (24). «Elle aurait sans doute su être lucide et bonne si on ne l'avait pas tant trompée. Elle se détestait, détestait les fausses certitudes sur lesquels sa mère lui avait laissé bâtir sa vie [...] » (I34). Chez Flaubert : « Avant qu'elle [Emma] se mariât, elle avait cru avoir de l'amour ; mais le bonheur qui aurait dû résulter de cet amour n'étant pas venu, il fallait qu'elle se fût trompée, songeait-elle. Et Emma cherchait à savoir ce que l'on entendait au juste dans la vie par les mots de félicité, de passion et d'ivresse, qui lui avaient paru si beaux dans les livres » (94). «Elles [les journées] allaient donc maintenant se suivre ainsi à la file, toujours pareilles, innombrables, et n'apportant rien! Les autres existences, si plates qu'elles fussent, avaient du moins la chance d'un événement. Une aventure amenait parfois des péripéties à l'infini, et le décor changeait. Mais, pour elle, rien n'arrivait, Dieu l'avait voulu ! L'avenir était un corridor tout noir, et qui avait au fond sa porte bien fermée » (I23-I24).

5 Voir également, ici même, la contribution d'Ariane de Testa, pp. 47-54. 
Ça se passe bien entre Richard et toi [lui disait sa mère]. Ce n'était pas une interrogation, plutôt une sommation, sa mère ne lui laissait aucune chance de discuter le bonheur de cette vie de maman dont il avait été question depuis toujours, dans un monde d'abstraction dont Alissa s'était accommodée sans s'inquiéter ni se douter de rien, d'aucune vérité. Richard avait du charme, il était gai, il était entreprenant, il l'aimait, Alissa avait toujours désiré être la première à se marier, elle avait voulu un bébé et avait été tellement fière du dévouement dont il avait fait preuve pendant la grossesse. Pourtant, tout en elle se figeait d'anxiété à l'idée de s'être trop précipitée, de n'être plus sûre de l'aimer. De tout cela pourtant, rien ne pouvait être avoué. Le temps du choix était passé, mais avait-elle seulement choisi ? Ne s'était-elle pas simplement conformée à l'incroyable béguin de sa mère pour Richard, à la légende de leur couple, qu'on disait le plus sexy du campus, à l'envoûtement de la grande photo prise par Audrey six ans plus tôt sur la plage de Santa Monica : lui la couvant de ses épaules rousses, ses dents écartées mordant son oreille, elle appuyée sur ses coudes, ses mains en corolle soutenant son visage doré au nez un peu long, aux doubles fossettes prolongeant la pulpe claire et luisante de son sourire (30).

À la vie « abstraite », loin de toute « vérité », vient se confronter la vraie vie, concrète, la « légende » de leur couple cède la place à la réalité vécue au quotidien. Le « monde indolore de la rue Denslow » (65), où Alissa a grandi, entourée de " promesses et de certitude chuchotées » (65) par sa mère, se voit remplacé par un monde marqué de doutes et de souffrances. Face à cette nouvelle réalité, Alissa est constamment tentée de régresser, tantôt en se gavant de sucré, tantôt en se réfugiant dans des crises de tétanie ${ }^{6}$. Mais, rien n'y fait : l'accès au « monde hypnotisant d'avant le réveil » (32) lui reste définitivement interdit. Una est là, implacablement, présence pure qui attend d'être prise en charge.

Le deuxième volet, social et politique, se met en place autour du personnage de Jim, jeune vétéran rentré gravement mutilé de son service militaire en Irak. Lors d'une fête de bienvenue organisée pour son retour par son épouse et ses copains, Alissa observe l'apparition de Jim. Les réflexions qui traversent son esprit à ce moment ne sont pas sans rappeler celles que lui inspire la présence du corps d'Una, dans son « inguérissable fragilité, avide, perdue, souffreteuse, incompréhensible» (22):

Un des invités avait poussé un cri de bienvenue qui fut relayé par quelquesuns et déclencha des rires dans le petit groupe qui avançait au rythme de Jim. Par défi ou courage, ou par une sorte de légitime cruauté envers eux, il s'était habillé d'un grand T-Shirt de sport jaune et d'un short d'où émer-

6 Le bref séjour à l'hôpital rendu nécessaire par un épisode fiévreux d'Una, peut également se comprendre dans le sens d'une régression, enlevant pour un moment la " responsabilité » (IOI) à Alissa. 
geait la rigidité mécanique, squelettique, presque cocasse, de son bras et de sa jambe en tiges de métal. [...] On les devinait tous gagnés par la griserie du dévouement et de leur maîtrise face à l'horreur contre laquelle leur conscience semblait avoir été anesthésiée. C'était comme s'il fallait à tout prix saouler Jim de sa propre folie à nier la monstrueuse réalité. C'était vertigineux d'insouciance (55).

On retrouve, dans la perception de cette scène par Alissa, la mise à nu des mêmes mécanismes que ceux qui régissent sa propre vie avant la naissance d'Una : la « conscience anesthésiée », la négation de la réalité dite " monstrueuse », une « insouciance vertigineuse » face à la gravité des évènements. Mais, cette fois-ci, c'est toute la société qui semble affectée d'un état d'aveuglement, sous couvert, en l'occurrence, d'une attitude de 'faux dévouement' vis-à-vis de Jim. Tout le monde ou presque joue au jeu du héros, entre ignorance, désinformation et idéalisation. Il n'y a qu'Yvan, un voisin d'Alissa et de Richard, qui se prononce de façon critique sur cette guerre, dans laquelle les jeunes ne feraient que «bousiller leur vie » (I07), et la mère de Jim, qui refuse toute complicité :

\begin{abstract}
Alissa apprit par Kathy qu'elle [la mère de Jim] avait fait paraître dans un quotidien une lettre véhémente expliquant pourquoi elle ne pardonnerait jamais, ni à Dieu ni aux hommes, de faire vivre son fils dans cet état. Il avait fallu toute la persuasion des copains pour la convaincre de venir. Alissa souffla un petit rire incrédule. Mais qui étaient-ils donc pour obliger cette femme à assister à ça ? (56)
\end{abstract}

Et puis, il y a Alissa, en train de se réveiller, comme le montrent les citations précédentes; et, finalement, il y a Jim lui-même, de plus en plus « lucide » (59) lui aussi, et qui, ne supportant plus l'hypocrisie de la société et des gens qui l'entourent, essaie d'abord de tuer son épouse pour ensuite se donner la mort et mettre ainsi un terme au drame. L'attitude de Richard et de ses copains, en revanche, est plus ambiguë, comme le montre à lui seul le tatouage qu'ils se font faire : " une petite tête d'aigle d'où gouttaient des larmes ou du sang »(I25). S’il est difficile de ne pas lire la figure de « l'aigle» comme renvoyant à l'insigne des SEALs, le fait qu'il y ait des larmes et du sang coulant de la tête du rapace, semble en même temps désidéaliser, désacraliser le symbole et, partant, les valeurs de patriotisme et d'héroïsme qui y sont attachées. Les films sur la guerre en Irak tournés par les soldats que ne cessent de regarder Richard et ses amis, ces « images hypnotisantes d'interminable désert » (IOI) où « il ne se passe quasiment rien » (98), ne peuvent-ils être compris, eux-aussi, comme une expression de l'anesthésie collective ? Si Alissa s'est réveillée de son état d'" insouciante amnésie au monde qu'avait été sa jeunesse » (IO5), incapable désormais de ne plus regar- 
der la réalité et la vérité en face - notons que deux corps sont à l'origine de son expulsion du monde idéalisé : le corps parfait d'Una et le corps mutilé de Jim -, le réveil de la société semble loin encore.

L'Implacable Brutalité du réveil peut être lu comme un texte qui montre les difficultés et la douloureuse nécessité de devenir adulte, si l'on comprend par ce terme, au-delà du passage de l'adolescence (retardée) à l'âge adulte, la capacité de reconnaître et d'admettre ce qui est, d'assumer la vérité, celle sur le monde et celle sur soi-même, de réaliser que chaque acte marque la réalité, et que, dans ce sens, la vie est toujours, à tout moment, définitive, ne souffrant pas de répétition. Si cette idée rappelle l'existentialisme sartrien et Alissa a bien « un sentiment d'absurdité » (54) quand elle voit apparaître le corps mutilé de Jim -, on est cependant très loin ici de toute idée d'un roman à thèse. L'art de Pascale Kramer consiste à nous faire vivre des expériences douloureuses au rythme même de celles éprouvées par les personnages mis en scène et nous invite à en tirer une réflexion existentielle dont la responsabilité et la formulation nous incombent à nous seuls. Dans l'acte de lecture, nous devenons ainsi un peu plus adultes, un peu plus autonomes dans nos jugements. C'est là un aspect fondamentalement politique.

\section{Rester dans le vrai}

Autopsie d'un père est le livre le plus ouvertement politique, à ce jour, de Pascale Kramer. C'est l'histoire d'Ania et de son père Gabriel, ancien journaliste à la radio, qui, d'intellectuel de gauche, a évolué peu à peu vers une position réactionnaire. Après le meurtre d'un Comorien commis par deux jeunes du village proche de sa maison de campagne, il avait pris publiquement la défense de ces derniers, ce qui avait déclenché un scandale, au terme duquel il s'était vu « exclu de l'antenne, à la demande de l'ensemble de la rédaction » (Kramer $2016: 20)$. Le roman s'ouvre sur un «Prologue » dont la fin raconte le suicide de Gabriel.

Autopsie d'un père est aussi le premier livre de Pascale Kramer qui quitte le principe narratif de la focalisation interne sur un seul personnage : le « Prologue » est en effet écrit depuis la perspective de Gabriel, alors que le roman proprement dit, qui passe en revue la vie du père et de la fille, est construit dans celle d'Ania. Cette structure énonciative se verra élargie à une véritable polyphonie dans Une famille, qui donnera la voix à tour de rôle à cinq personnages différents.

Le choix narratif dans Autopsie d'un père nous fournit un premier élément important pour nos réflexions sur les présences du politique dans l'œuvre de Pascale Kramer : loin de le rejeter d'emblée dans une position objectale, définie par le seul regard des autres, le texte donne la voix à Gabriel lui aussi, lui accordant ainsi le statut de sujet, ayant droit au chapitre. Ceci non pas 
pour justifier ses opinions, mais pour en faire (entre)voir les raisons, ou au moins quelques éléments d'anamnèse. De manière performative, le roman, de par sa structure énonciative même, non seulement invite, mais oblige le lecteur à entrer en dialogue avec qui ne pense pas (nécessairement) comme lui.

Dans le «Prologue », nous accompagnons Gabriel lors d'un trajet en train, le soir même d'une visite inattendue de sa fille et de son petit-fils, Théo, qui, sans le savoir, se trouvent dans le même wagon que lui, trajet qui le conduit de sa maison de campagne à son petit appartement parisien. Ces premières pages du roman nous montrent un homme qui se sent seul et isolé, «banni » (Io) de la société, mais qui, en même temps, ne regrette pas la phrase qu'il avait prononcée après le meurtre, particulièrement brutal, du Comorien commis par les deux jeunes de la région, et qui avait provoqué le tollé général : "'La mort de cet homme, avait-il alors dit, me concerne moins que le sort de ces deux jeunes qui sont ici chez eux' " (53). Y repensant dans le train, Gabriel se fait la réflexion suivante : « Il aurait fallu pouvoir rire de lui-même, se dit-il, de n'avoir à ce point rien pressenti de l'isolement de banni auquel il s'était condamné, finalement en une seule phrase, que d'ailleurs il ne reniait pas (IO)».

Le trajet en train qui conduit Gabriel de la province à Paris peut être lu comme un condensé ou une scène miniature de ce que le roman développera plus en détail, et ceci sur deux plans, étroitement liés l'un à l'autre : celui de la relation entre le père et sa fille, et celui du rapport de Gabriel aux autres, à la société. Dans ce texte encore, Pascale Kramer entrelace un drame familial et un drame social.

Dans le wagon, Gabriel est en position d'observateur et de raisonneur. Il regarde sa fille et son petit-fils, qui, eux, rappelons-le, ne le voient pas, et fait d'eux l'objet de ses considérations. Si l'idée de se manifester l'effleure un court instant, il la rejette pourtant aussitôt : « que trouveraient-ils de plus à se dire ? » (II). Le refus d'un rapport vivant, physique, aux gens qui transparaît ici et qui fait que Gabriel ne voit les autres qu'en tant que surface de projection, constitue précisément ce dont sa fille a souffert tout au long de sa vie, comme nous l'apprendrons dans le roman proprement dit, par la voix d'Ania elle-même?

Ce n'est pas seulement vis-à-vis de sa fille et de son petit-fils que Gabriel se met en position d'observateur et de raisonneur, mais également vis-à-vis des autres passagers qui l'entourent. Le wagon du train devient lisible à cet égard comme un espace métonymique de la société française, telle que perçue par Gabriel. Celui-ci y souffre de ce qu'il ressent comme une "promiscuité » (I5) oppressante et envahissante, et sans que le texte le dise, on est

7 Voir également, ici même, l'article de Ruth Gantert, pp. 55-59. 
amené à comprendre ce terme, ainsi que la métaphore des « cargaisons dépareillées, mal élevées » dans la citation suivante, dans un sens xénophobe sinon raciste : «Se serait-il fait lyncher si ces gens avaient su ce qu'il ne se gênait plus de dire à leur sujet : ces cargaisons dépareillées, mal élevées, avec lesquelles il fallait voyager désormais ? (I4). À aucun moment, Gabriel n'est tenté d'entrer en contact avec les personnes qu'il observe, pas plus qu'avec Ania et Théo. Il reste dans ses propres idées, dans son monde tout d'abstraction. En même temps, il revendique pour lui-même une position de supériorité intellectuelle, dans ce pays « déserté, selon lui, par l'exigence et par l'esprit » (I4), et aussi de sincérité, taxant ceux qui ne partagent pas ses idées d'« hypocrites » et de «naïfs » (I2). Plus loin dans le roman, Clara, la dernière compagne de Gabriel, parlera d'« idéalistes ", " avec un mépris formidable » (I59), pour désigner les gens qui continuent à penser comme Gabriel lui-même avait pensé avant son revirement idéologique. N'y a-t-il pas un souffle de La Nausée qui passe ici ? Les "idéalistes » ne peuvent-ils être lus en référence à l'« Autodidacte » et à tous les « humanistes » décriés par Roquentin (Sartre I938 : I67-I68) ? Tout comme ce dernier, Gabriel est pris de «nausée » (I6) face au monde qui l'entoure, et tout comme Roquentin encore, il note ses expériences dans un journal ; il y a jusqu'aux marronniers (I5) qui sont présents. Mais en quoi consisterait alors 'l'illumination' de Gabriel, qui, contrairement à Roquentin, seulement effleuré, celui-ci, par l'idée de se supprimer, le poussera au suicide?

L'état de proscrit où il se trouvait depuis plusieurs semaines lui causait un mal-être auquel la fatigue et la distance le rendaient plus lucide, nota-t-il dans son journal, en préambule à plusieurs pages sur la sensation d'oppressement qui lui avait rendu la maison hostile et son isolement soudain si magistral en l'absence de Clara (I6).

'L'illumination' de Gabriel ne concerne pas la position de l'homme dans le monde, comme c'est le cas de celle dont nous fait part Roquentin ; elle est liée uniquement à son propre sort, à la vision de sa propre situation d'« isolement magistral » qui s'offre soudainement à lui. Ce sont la reconnaissance de l'abîme désormais infranchissable qui le sépare des autres et celle de son propre statut de paria aux yeux de la société qui semblent provoquer le suicide. En s'ingurgitant des éclats d'un verre à moutarde, Gabriel se fait taire en avalant des objets aussi tranchants que furent ses propres paroles. Cri accusateur lancé à la société ou acte d'ultime assomption de ses opinions ? Rien ne nous indique qu'il faille trancher entre ces deux interprétations.

Les éléments mis en place dans le « Prologue »sont repris et développés dans le roman proprement dit, qui adopte la perspective de la fille. Le centre de gravitation du texte, tant en ce qui concerne l'action relatée après la mort 
de Gabriel que pour ce qui est des souvenirs d'enfance d'Ania, est la maison de campagne, au nom évocateur des «Épinettes », où celle-ci a grandi avec son père.

Tout comme le wagon du train, ce lieu peut être compris comme une figuration spatiale de l'isolement de Gabriel dans un monde d'abstraction, protégé par une 'haie d'épines'. Or ce monde d'abstraction n'est pas dans un rapport exclusif avec le revirement idéologique de Gabriel ; tout au contraire, il est l'expression d'un principe qui régit l'auto-construction du personnage dès sa jeunesse. Comme nous l'apprenons de la bouche de Clara vers la fin du texte, Gabriel s'était forgé pendant ses études une image précise de lui-même - celle d'un homme cultivé et, pouvons-nous ajouter, d'un intellectuel de gauche, ouvert à d'autres cultures, époux d'une femme iranienne - qui ne pouvait se réaliser que dans «l'effacement » total de ses origines : «Il [Gabriel] a tellement travaillé pour effacer toute rusticité en lui et atteindre à un raffinement de connaissance dont il est vrai que tout le monde se moque aujourd'hui » (I69). Cette auto-image construite au moyen d'immenses efforts et au prix de la négation du monde réel, ne pouvait se maintenir que dans la distance vis-à-vis de l'autre, dont la présence risque en effet à chaque moment d'ébranler une identité si péniblement acquise. Ainsi, pendant toute son enfance, Ania « n'avait jamais vu Gabriel fréquenter qui que ce soit du village, tout au plus s'y était-il intéressé de façon théorique » (I28).

L'évolution de ses idées politiques ne semble guère affecter l'être-dansle-monde de Gabriel, qui continue à vivre dans un univers largement abstrait. S'il est vrai qu'il a rendu visite aux deux jeunes assassins en prison, rien ne nous dit qu'il ait cherché à entrer véritablement en contact avec des personnes appartenant aux couches sociales avec lesquelles il s'était solidarisé en pensées et en paroles. Avec Chloé, par exemple, une ancienne copine de classe d'Ania, dont la mère « obèse » (29) dormait à l'époque «tout le jour au milieu d'un élevage d'oiseaux », et qui vit maintenant tout près des Épinettes, «dans un chaos un peu perdu », avec une " grosse fillette de plusieurs mois », alors que « les trois aînés avaient été placés » (30). Quand Gabriel pense à Chloé dans le train, il ne revoit qu'une «fillette un peu sale, aux yeux très pâles, venue accompagnée de sa mère, il y avait au moins trente ans, à un anniversaire » (II). Le fait qu'il perçoive le monde réel à travers un écran se voit figurativisé dans le passage qui clôt le "Prologue ». Regardant par la vitre du train qui le conduit à Paris, Gabriel croit voir un homme résolu à se tuer. Dans le dernier message qu'il laisse sur le portable de Clara qu'il a essayé de joindre en vain, il revient sur cette scène, précisément, et ce sont là ses derniers mots en même temps que les dernières lignes $\mathrm{du}$ « Prologue» : 
Avant de raccrocher, il ajouta encore qu'il avait cru voir un type se jeter sur les voies, un ouvrier ou un cheminot, un homme d'un monde de labeur qui avait été celui de ses parents, un monde révolu, discrédité, dont la nostalgie ne serait jamais acceptable ni supportable (I6-I7).

C'est par une vitre et à distance qu'il voit l'autre, ou, plutôt, qu'il ne le voit pas, mais le transforme en surface de projection de ses propres idées, de ses propres douleurs. Ses souffrances, réelles, relèvent alors moins de l'empathie, portée vers l'autre, que d'une sentimentalité qui n'a pour point de référence que sa propre personne.

L'abstraction ainsi entendue semble aussi le trait dominant du rapport que Gabriel a entretenu tout au long des années avec sa fille. Selon Ania, «il n'était en fait jamais vraiment entré dans le concret de son rôle de père » (I20). Après la mort de sa mère, quand il vivait seul avec sa fille, le « couple qu'ils formaient et que Gabriel adorait, le veuf et la petite fille, avait besoin du décor des Épinettes " (49), comme pour se conformer à un tableau idéal et pathétique. La mise en scène d'une posture se prolonge jusqu'à la fin de sa vie. Se rappelant sa dernière visite chez son père, le jour même de son suicide, Ania

[...] revoyait Gabriel, assis déhanché dans le fauteuil en osier, exposant ses projets pour occuper la liberté dont il allait jouir maintenant [après avoir perdu son poste à la radio], se montrant en quelque sorte satisfait du scandale provoqué et s'amusant de ce qu'elle pouvait penser. C'était fou d'être à ce point dans la dérobade et le jeu (36).

Face au corps mort, elle adopte, cyniquement, le point de vue du paraître qui avait toujours été celui de son père : « La mort ne vous avantage pas, songea-t-elle. Gabriel n'avait pas dû préméditer la sienne, il l'aurait mieux mise en scène » (50). Tout comme Alissa dans L'Implacable Brutalité du réveil, Gabriel vit, lui aussi, dans un monde fait de scénarios. Et tout comme Alissa encore, il semble tenté de régresser face à la complexité du monde réel qu'il arrive de moins en moins à garder à distance : en effet, ne pourrait-on pas comprendre dans ce sens aussi son revirement idéologique, qui, selon Clara, l'avait rapproché « des sentiments plus traditionnels » (74), ceux, donc, probablement, de ses origines ? Le dernier message laissé à sa compagne que nous avons cité plus haut nous confirmerait dans cette interprétation, tout comme le besoin existentiel, enfantin presque, que Gabriel a de Clara. Mais, alors qu'Alissa, douloureusement réveillée, semble finalement prête à se confronter à la vie, Gabriel choisit de mourir.

Le fait que Gabriel vive largement dans un monde d'abstraction n'exclut pas des moments où il semble montrer des réactions qu'on qualifiera bien d'empathiques, ainsi, par exemple, quand il fait tout pour déculpabiliser sa 
fille d'avoir donné naissance à un enfant sourd. Mais c'est comme s'il lui était impossible d'assumer pour de bon ces élans vers l'autre, de les vivre réellement : «C'était donc toujours ainsi qu'il s'était vu, et qu'il avait toujours été finalement », conclut Ania : « affranchi jusqu'à l'inacceptable des pitiés et des conventions » (I66).

Si le roman montre les failles de la position réactionnaire de Gabriel, ses anciens amis, restés fidèles à leurs idées de gauche, et qui l'ont tous abandonné avec horreur et indignation, n'en sortent pas indemnes. Quand Ania se rappelle les anciens « convives du dimanche », elle les voit, eux aussi, dans une posture de « décontraction condescendante avec le village » (20). Clara, à son tour, met le doigt sur leur hypocrisie quand elle mentionne qu'aucun d'eux ne s'était soucié le moins du monde de ce qui était arrivé au corps du Comorien après son meurtre. Dans une lettre de condoléance adressée à Ania, une des anciennes amies de Gabriel, Kathy, fournit une analyse lucide de son propre comportement :

La lettre restée sur la table était d'une certaine Kathy qui disait l'avoir [i.e. Ania] croisée quelques fois aux Épinettes. Elle racontait avoir participé à la cabale contre Gabriel qu'elle qualifiait elle-même d'assassine dans une sorte de confession où elle expliquait [...] le sentiment rassurant de faire partie d'un corps, l'impression, après coup, que tout cela avait été d'une facilité sans courage (II5).

La teneur de cette lettre peut être rapprochée des propos de Pascale Kramer au sujet de l'« affaire Millet » :

[...] pour Autopsie d'un père, c'était la montée de ce qu'on appelle les nouveaux réacs. Puis il y a eu l'affaire Richard Millet et son Éloge littéraire d'Anders Brei$v i k$, jeune néonazi qui a commis un massacre en Norvège. Millet a été complètement mis au ban de la société. Nous avons tous signé une lettre ouverte parue dans Le Monde, initiée par Annie Ernaux. Je n'en ai pas été très fière, nous ne faisions que tirer sur l'ambulance. Et puis j'avais connu Richard Millet, un homme instruit et intelligent, et je me demandais ce qui l'avait amené à écrire des choses comme ça. J'ai eu envie d'appréhender ça aussi à travers un roman ${ }^{8}$.

Contrairement à son père, Ania ne cesse de se frotter à la réalité. Elle n'a pu vivre, c'est-à-dire développer sa propre identité, qu'en quittant les Épinettes, espace clos dans lequel, après la mort de sa mère, de manière à peine paradoxale, elle ne s'était sentie bien que malade, éprouvant « la sensation rêche des draps propres sur sa peau brûlante »(II4). La 'haie d'épines'

8 Voir l'entretien, ici même, p. 95-I97. 
qui avait protégé Gabriel de l'extérieur, avait enfermé Ania à l'intérieur, telle une cage. Encore adolescente, elle a hâte de quitter le 'centre du monde' de son père pour vivre dans les 'périphéries', en banlieue - la géométrie des lieux a une importance capitale dans tous les romans de Pascale Kramer - : elle épouse un homme serbe de religion musulmane qu'elle quitte au bout de quelques années, elle accouche d'un enfant sourd dont elle s'occupe pratiquement seule avec tout l'amour et toute l'attention dont elle est capable, elle travaille dans une crèche où elle garde des enfants d'immigrés, venant de milieux défavorisés. Ania n'est pas exempte de doutes et de peurs, d'inquiétudes et de colères, tout au contraire, et elle connaît, elle aussi, des réflexes xénophobes. Après avoir observé un homme au visage "de nuit luisante " (IO4), père d'Issa, un petit garçon dont elle s'occupe à la crèche, inspecter un vieux matelas destiné au rebut, elle découvre en elle des sentiments qu'il lui est difficile et douloureux d'avouer :

Cette brève scène lui laissa toute la matinée une impression de gêne et d'inconfort. [...] La préoccupation de ces vies si précaires, échouées là de plus en plus nombreuses, l'accablait d'une angoisse et de réactions de rejet qu'elle n’arrivait pas toujours à raisonner (IO4).

Elle se découvre aussi, presque malgré elle, un « sentiment d'appartenance de classe » (I29). Mais, contrairement à son père, elle admet ses réactions dans tout ce qu'elles ont de perturbant, de honteux, aussi, et essaie de contrôler ses premières impulsions, étant consciente du danger destructeur qui leur est inhérent:

Jamais elle ne se serait avoué qu'elle non plus n'aimait pas cette femme, la mère d'Issa. La haine prenait ici avec une fureur jubilatoire d'incendie. Ania restait au bord des siennes. Ce genre de remarques [" on ne les oblige pas à rester ", disait une de ses collègues], devenues tellement banales désormais dans le quartier, ouvrait des gouffres en elle. Quelle insouciance à ses propres peurs et violences fallait-il pour risquer ainsi l'embrasement ? (Io6)

Elle, qui s'était trouvée en échec scolaire permanent, elle, taxée d'analphabète par son propre père (48), elle, qui s'était sentie " tellement déficiente, étant gamine » dans ces « milieux intouchables et cultivés » (24) dans lesquels circulaient Gabriel et ses amis, semble bien la plus cultivée, humainement, de tous les personnages du roman. Jamais sûre de sa propre position, attentive et perméable aux moindres émotions et réactions d'autrui, elle reste interdite devant la violence croisée des deux camps figés qui s'affrontent après la mort de son père. Le roman se termine par l'incendie d'une maison avoisinant les Épinettes, habitée par un couple d'« idéalistes », 
mise à feu par des jeunes du village pour venger la profanation du tombeau de Gabriel :

Ania, et ce sont les dernières lignes du roman, resta plantée là à les dévisager. Elle n'arrivait pas à croire que ce couple avait à voir avec le saccage de la tombe. Et elle ne comprenait pas davantage de quelle insondable rancune les jeunes types, si veules et lents hier à s'avancer jusqu'à la tombe, avaient tiré l'énergie d'un acte d'une telle violence (I73).

Autopsie d'un père est un roman politique, moins, peut-être, parce qu'il parle de politique, ce qu'il fait aussi, que, bien plus, parce qu'il nous montre les conditions mêmes d'une bonne politique, comme on parle, dans le sens aristotélicien, d'une bonne vie. Il déploie les dangers de l'abstraction inhérents à toute position idéologique qui refuse de voir l'autre dans sa différence à chaque fois singulière et le monde tel qu'il est, base même, en effet, de toute action raisonnable dirigée vers un futur vivable. Ce faisant, il montre la nécessité d'une position de l'entre-deux - et le fait qu'Ania, seul personnage capable de tenir cette position, soit une métisse n'est peut-être pas tout à fait insignifiant dans ce contexte -, rejoignant, par là, les propos de Pascale Kramer elle-même, dans sa contribution sur le Point de vue suisse de Carl Spitteler :

[...] c'est la position la plus difficile à tenir : reconsidérer ses certitudes, entendre les causes et bonnes raisons de l'autre, accepter ses torts... toute cette besogne sans panache qui consiste à travailler à la paix pour ne pas avoir à la faire sur les décombres des deux camps (Kramer 2019:52).

Et, plus loin : « [...] la fracture se creuse irrémédiablement. Peut-être eston arrivés au bout des dernières 'pauses digestives et somnolences qu'on appelle la paix', comme le dit tristement Spitteler » (59). Ce n'est qu'en dépassant cette fracture que nous pourrons avancer, en nous con-frontant, ensemble, donc, à la réalité qui ne cesse et ne cessera pas de changer.

Le roman peut être dit politique dans un autre sens encore, méta-littéraire. George Steiner, dont on connaît le scepticisme concernant la valeur morale de la littérature et de l'art en général, s'exprimait ainsi, en 2000, dans un entretien accordé à L'Express :

J'ai commencé, dès l'enfance, à me laisser prendre par le vertige de l'abstraction. Il faut que je le dise : quand j'ai passé ma journée à étudier Le Roi Lear ou Les Fleurs du mal et que je rentre le soir encore sous l'emprise de cette transcendance, eh bien, je n'entends pas le cri dans la rue. Il y a dans la haute culture une force telle que les vraies misères humaines, banales, vulgaires, chaotiques, ont moins d'impact. La larme de Cordélia est plus vive, 
plus immédiate, plus réelle que le cri dans la rue. L'esthétique, la beauté, une page de Shakespeare, de Kant, de Descartes, de Hegel ou de Bergson, font battre en retraite un peu de la réalité quotidienne. Alors, après avoir enseigné pendant cinquante-deux ans, je me demande maintenant : «Est-ce que je savais ce que je faisais? Pouvons-nous vraiment établir un lien entre la haute culture et une conduite plus humaine? » Je me pose sans cesse cette question (Steiner 2000).

Montrant, au travers de la narration, les dangers de l'abstraction et nous mettant, en même temps, en contact avec le concret du vécu des personnages, Autopsie d'un père devient, sur un plan performatif, un livre qui (re) donne confiance dans le potentiel humaniste de l'art, entendu comme un frottement à l'humain, comme une sollicitation à entrer dans le vif, là surtout, où cela fait mal.

Écriture implacable que celle de Pascale Kramer : non seulement parce qu'elle nous expose de manière inexorable aux souffrances des autres, qui deviennent les nôtres, mais aussi, parce que, sans nous donner les clefs, elle nous ouvre des portes, nous obligeant constamment à rester dans le vrai, quoi qu'il advienne et quoi qu'il nous en coûte.

\section{Bibliographie}

Bähler, Ursula, « Retour à l'homme : Marie N'Diaye et Pascale Kramer », Un retour des normes romanesques dans la littérature française contemporaine, éd. Wolfgang Asholt \& Marc Dambre, Paris, Presses Sorbonne Nouvelle, 20II, pp. 293-3IO.

—. \& Klinkert, Thomas, "Avant-propos ", À quoi bon l'enseignement de la littérature ?, éd. Ursula Bähler \& Thomas Klinkert, Versants, 63/I, 2016, pp. 7-I2, en ligne : https://bop.unibe.ch/versants/article/view/355I/5546, (consulté le 20/02/2020).

—. \& Fröhlicher, Peter, « Avant-propos », À quoi bon la littérature ? Réponses à travers les siècles, de Rabelais à Bonnefoy, éd. Ursula Bähler, Peter Fröhlicher \& Reto Zöllner, Paris, Garnier, 2019, pp. 7-I6.

Berset, Alain, Prix suisses de littérature 20I7, Allocution du Conseiller fédéral Alain Berset à l'occasion des Prix suisses de littérature, Berne, I6/02/2017, en ligne : https://www.edi.admin.ch/edi/fr/home/documentation/discours.msg-id-65676.html (consulté le 20/02/2020).

Flaubert, Gustave, Madame Bovary, éd. B. Ajac, Paris, Flammarion, 2006.

Kramer, Pascale, Retour d'Uruguay, Paris, Mercure de France, 2003.

-. L'Implacable Brutalité du réveil, Paris, Mercure de France, 2009.

-. Un homme ébranlé, Paris, Mercure de France, 2011.

-. Autopsie d'un père, Paris, Flammarion, 2016. 
-. Chronique d'un lieu en partage, Ivry-sur-Seine, Éditions de l'Atelier/Éditions ouvrières, 2016.

—. «Salauds de pauvres ! L'effroi et la honte», LeIhebdo, 2 novembre 20I6, en ligne : https://leIhebdo.fr/journal/salauds-de-pauvres/I28/article/l-effroiet-la-honte-I897.html (consulté le 20/02/2020).

—. "Une éternelle étrangère en France », Helvétique équilibre. Dialogues avec le Point de vue suisse du Prix Nobel de littérature 1919, éd. C. Luscher, Genève, Zoé, 20I9, pp. 5I-59.

Sartre, Jean-Paul, La Nausée, Paris, Gallimard, 1938.

Steiner, George, "La culture ne rend pas plus humain », propos recueillis par Dominique Simonnet, L'Express, 28 décembre 2000, en ligne : https://www.lexpress.fr/culture/livre/la-culture-ne-rend-pas-plus-humain_8I7466.html (consulté le 20/02/2020). 
